\begin{tabular}{c} 
Brazilian Journal \\
of Chemical \\
Engineering \\
\hline
\end{tabular}

ISSN 0104-6632

Printed in Brazil

www.abeq.org.br/bjche

Vol. 32, No. 04, pp. 895 - 901, October - December, 2015

dx.doi.org/10.1590/0104-6632.20150324s20140142

\title{
REMOVAL OF WATER CONTENT FROM BIODIESEL AND DIESEL FUEL USING HYDROGEL ADSORBENTS
}

\author{
P. B. L. Fregolente ${ }^{1,2^{*}}$, M. R. Wolf Maciel $^{1}$ and L. S. Oliveira ${ }^{2}$ \\ ${ }^{1}$ Separation Process Development Laboratory, Chemical Engineering School, \\ State University of Campinas, 13083-852, Campinas - SP, Brazil. \\ ${ }^{2}$ Laboratory of Material Science, Salesian University Center of São Paulo, \\ 13087-290, Campinas - SP, Brazil. \\ E-mail: patriciafregolente@yahoo.com.br
}

(Submitted: October 31, 2014 ; Revised: February 5, 2015 ; Accepted: February 8, 2015)

\begin{abstract}
The removal of water content present in fuels such as biodiesel and diesel is quite important to adequate the fuel to standards for commercialization and to avoid corrosion of storage tanks and injection equipment in diesel engines. In this study, hydrophilic hydrogels were employed to remove the water content in biodiesel and diesel fuel samples. The results showed that the hydrogels were capable of decreasing the free water content and also the soluble water content present in the samples. The highest decrease of total water content in samples of biodiesel was $53.3 \% \mathrm{wt}$ and for diesel samples the reduction of water content was $32.0 \%$, starting with samples that had $2160 \mathrm{ppm}$ and $240 \mathrm{ppm}$ of water, respectively. The highest decrease of total water content (free and soluble water) for diesel samples was $80.4 \% \mathrm{wt}$, from a diesel sample initially containing 348 ppm of total water content.

Keywords: Water content; Biodiesel; Diesel.
\end{abstract}

\section{INTRODUCTION}

Fuels can incorporate water during the production process, transportation and storage (He et al., 2007; Shah et al., 2010). Water in fuels has always been a problem, promoting severe damage on fuel-injection equipment and/or contributing to corrosion in storage tanks. The water content in fuels can be classified as free water and soluble water. Water solubility depends on the temperature and also on the composition of the fuel. However, the hygroscopic nature of biodiesel can lead to an increase of soluble water content during production and storage (Fregolente $e t$ al., 2010; Oliveira et al., 2008). During biodiesel production, water is used to remove catalyst, soap and traces of glycerol. The remaining water content must be removed through an evaporator or a vacuum drier.
Finally, biodiesel is stored and distributed to be sold (Atadashi et al., 2012; Ferella et al., 2010). Once stored, biodiesel can absorb more humidity than petroleum diesel since fatty acid methyl esters (FAMEs) are hygroscopic compounds, making biodiesel much more hydrophilic than regular diesel (He et al., 2007). Biodiesel clearly offers environmental, commercial and performance benefits, since biodiesel does not contain sulfur, aromatic hydrocarbons, metals or crude oil residues. However, it must be emphasized that biofuel's performance heavily depends on the purity of the final product and the complete absence of particulates or contaminants, e.g., water (Oliveira $e t$ al., 2008).

The free water content in biodiesel and diesel fuel promotes biological growth in storage tanks, which could lead to corrosion of metals (copper, iron, steel

*To whom correspondence should be addressed 
and others) and formation of sludge and slime, thereby causing blockage of fuel filters and fuel lines, which could in turn damage vehicle fuel injection systems (He et al., 2007; ASTM, 2003; Fazal et al., 2013; Bücker et al., 2011; Sørensen et al., 2011). Free or emulsified water can cause turbidity, mainly in diesel fuel, making diesel fuel out of specification for its commercialization. Similarly, condensation and precipitation of water in the fuels may occur during storage and transportation.

In fact, the removal of water from fuels is difficult - or indeed not possible. The water removal techniques used for diesel fuel are vacuum drying, treatment with salt and the use of coalescing filters (Leung et al., 2006). For water removal during biodiesel production energy-expensive processes such as heating or a vacuum flash are used (Atadashi and Aroua, 2012). Few studies aim to develop techniques of detection of water content in biodiesel. Bampi et al. (2013) determined experimentally the water content and average droplet size of emulsions prepared from industrial biodiesel using infrared spectroscopy. The maximum amount of allowed water content in biodiesel as specified in ASTM standard D6751 is 500 ppm (ASTM, 2003). If during the storage biodiesel or diesel absorb water from the ambient, a reprocessing is required, causing losses to the manufacturers.

Knowledge of the water content present in biodiesel and diesel is quite important and very little information is available on this aspect. This research employs dry polymeric hydrogel for removal of the water content of diesel and biodiesel samples. Based on the advantages of the hydrophilicity that hydrogels present, swelling in the presence of water, we have investigated the use of these polymeric materials for water removal from fuels.

Hydrogels are three-dimensional networks of polymer chains that can adsorb and/or retain a large amount of water inside. The extent of swelling is a unique property of hydrogel particles. Their properties make them useful in a variety of fields, including biomedical and pharmaceutical applications, oil recovery, molecular separation, environment-sensitive display devices, and other applications (Nur et al., 2009; Qunwei et al., 2008; Bassil et al., 2008; Deligkaris et al., 2010). Nur et al. (2008) used different microgels of PNIPAAm for removal of the water content in biodiesel samples. From samples containing initially $1800 \mathrm{ppm}$ of water, the use of microgels reduced the water content of the samples to less than 500 ppm. In a previous work, Fregolente et al. (2012) synthesized a hydrogel of polyacrylamide and the dry particles were used to remove the water content of saturated biodiesel and diesel fuel samples. A reduction of $65 \%$ in the water content was verified in biodiesel samples.

\section{MATERIALS AND METHODS}

\section{Materials}

All the experiments were performed employing fuel samples of typical commercial Brazilian biodiesel and S10 diesel fuel ( $<10 \mathrm{mg} / \mathrm{kg}$ sulphur), obtained from Petrobras-BR. Hydranal Solvent Oil and Hydranal Titrant (Sigma-Aldrich) were used for Karl Fischer analyses. The synthesis of hydrogels used acrylamide (AAm) 98\% (Sigma-Aldrich), $N, N^{\prime}$ methylenebisacrylamide (MBAAm) 99\% (SigmaAldrich), and potassium persulfate 99\% (SigmaAldrich). The chemical compositions of the diesel and biodiesel samples are presented in Tables 1 and 2, respectively.

Table 1: Physical and Chemical Properties of Diesel Fuel $^{\mathbf{a}}$

\begin{tabular}{|c|c|c|}
\hline Hydrocarbon & $\boldsymbol{x}$ & $\mathbf{T}^{\mathbf{b}} /{ }^{\mathbf{o}} \mathbf{C}$ \\
\hline C8 - C10 & 0.11 & 196 \\
C11 & 0.11 & 216 \\
C12 & 0.16 & 254 \\
C15 & 0.17 & 271 \\
C16 & 0.08 & 287 \\
C18 & 0.10 & 314 \\
C20-C32 & 0.11 & $344-466$ \\
\hline
\end{tabular}

${ }^{\mathrm{a}}$ Density of S10 diesel oil $\rho=836.1 \mathrm{~kg} \cdot \mathrm{m}^{-3}$. ${ }^{\mathrm{b}}$ Boiling point. $x=$ mass fraction.

Table 2: Physical and Chemical Properties of Biodiesel Fuel ${ }^{\mathrm{a}}$

\begin{tabular}{|l|r|}
\hline Element & $\boldsymbol{x}$ \\
\hline total esters & 0.98 \\
esters from edible soybean & 0.90 \\
esters from beef tallow & 0.10 \\
glycerol & 0.15 \\
acylglycerols & 0.02 \\
free fatty acids & 0.004 \\
\hline
\end{tabular}

${ }^{\text {a }}$ Density of biodiesel $\rho=880.2 \mathrm{~kg} \cdot \mathrm{m}^{-3} \cdot x=$ mass fraction.

\section{Karl Fischer Measurement}

To evaluate the water content present in biodiesel and diesel fuel samples the volumetric Karl Fischer technique was used. The equipment used was a Titrando 841 Karl Fischer from Metrohm. All the analyses were done in triplicate. 


\section{Humidity Absorption}

Samples of biodiesel, diesel and blends from controlled humidity environments were measured for humidity absorption. Saturated chemical solutions of salts as calcium chloride hexahydrate $\left(\mathrm{CaCl}_{2} \cdot 6 \mathrm{H}_{2} \mathrm{O}\right)$, ammonium chloride $\left(\mathrm{NH}_{4} \mathrm{Cl}\right)$, sodium nitrite $\left(\mathrm{NaNO}_{2}\right)$ and sodium carbonate decahydrate $\left(\mathrm{Na}_{2} \mathrm{CO}_{3} \cdot 10 \mathrm{H}_{2} \mathrm{O}\right)$, with $35,66,79$ and $87 \%$ constant relative humidities, respectively, were prepared in desiccators (considered as a closed system) three days before the experiments. Samples were taken for water content measurements after 7 days under controlled atmosphere (Perry and Green, 1997). The relative humidity was measured employing a digital thermo-hygrometer Data Logger HT-4000. All experiments were done in duplicate.

\section{Saturated Water Content Curves for Biodiesel and Diesel Fuel}

Biodiesel and diesel fuel samples were saturated with water by mixing $70 \mathrm{~mL}$ of fuel sample with $70 \mathrm{~mL}$ of distilled water. The mixture was shaken vigorously for $1800 \mathrm{~s}$ using a magnetic agitator, to ensure proper contact between organic and aqueous phases and then allowed to settle for $24 \mathrm{~h}$. They were placed in controlled temperature of $10{ }^{\circ} \mathrm{C}, 20^{\circ} \mathrm{C}, 30^{\circ} \mathrm{C}, 40^{\circ} \mathrm{C}$ and $50^{\circ} \mathrm{C}$. After $24 \mathrm{~h}$ in controlled temperature, the fuel phase was centrifuged at $3000 \mathrm{rad} . \mathrm{s}^{-1}$ for $1200 \mathrm{~s}$ to eliminate micro water droplets in suspension and then the water content of the centrifuged samples was measured.

The ambient relative humidity $(\mathrm{RH})$ in all experiments was between 35 and $40 \%$, at $20{ }^{\circ} \mathrm{C}$. Measurements on samples of all blends were carried out in duplicate.

\section{Saturated Biodiesel-Diesel Fuel Samples}

Biodiesel and diesel fuels were saturated with water by mixing $70 \mathrm{~mL}$ of fuel samples with $70 \mathrm{~mL}$ of distilled water. The mixture was shaken vigorously for $30 \mathrm{~min}$ using a magnetic mixer to ensure proper contact between phases and then allowed to settle for 5 hours and not centrifuged. After this procedure, two separate phases were formed and the aqueous phase was removed. The organic phase (fuel phase) presenting a high content of water was used in experiments of removal of free water content. All results in the figures show the saturation line (dashed line). Above the dashed line, the samples (biodiesel or diesel) present both free water and soluble water content and below the dashed line the samples present only soluble water content.

\section{Unsaturated Biodiesel-Diesel Fuel Samples}

Samples of fuels taken for water content measurements after 10 days at 79 and $87 \%$ humidity content, described previously, were stored to be used in experiments of removal of soluble water content. According to previous work, the water content naturally absorbed by samples during this period of 10 days, inside the closed system at 79 and $87 \% \mathrm{RH}$, is considered to be part of the soluble water content (Fregolente et al., 2010).

\section{PAAm Hydrogel Synthesis}

The hydrogels of PAAm were synthesized by free radical polymerization, at room temperature. The acrylamide monomer (AAm) was mixed with $N, N^{\prime}-$ methylenebisacrylamide (MBAAm), in aqueous media. $5.0 \mathrm{wt} \%$ of AAm (in relation to the total water content), $2.5 \mathrm{wt} \%$ of MBAAm (in relation to the AAm wt \%) and $3.38 \mathrm{mmol} . \mathrm{L}^{-1}$ of potassium persulfate (PP) were dissolved in water and the solution was deoxygenated by bubbling with $\mathrm{N}_{2}$ during 10 min. Soon after, $3.21 \times 10^{-3}$ mol. $\mathrm{L}^{-1}$ of $N, N, N^{\prime}, N^{\prime}-$ tetramethylethylenediamine (TEMED) was added to this solution and the deoxygenation was maintained until the formation of gel. The system was kept at room temperature. The hydrogel formed was removed from the recipient, cut into smaller pieces and dehydrated in a heater at $70{ }^{\circ} \mathrm{C}$. The dehydrated hydrogels were used directly to remove water from biodiesel and diesel fuel samples.

\section{Swelling Measurements}

Each sample of hydrogel was placed in distilled water for swelling at room temperature. The swelling process was controlled by weighing. The ability to swell was expressed as the swelling ratio, W, via Eq. (1), in which $\mathrm{M}_{1}$ and $\mathrm{M}_{2}$ are the weights of the swollen and dry samples, respectively:

$$
W=\frac{M_{1}}{M_{2}}
$$

\section{Application and Recovery of Dry Hydrogel to Remove the Free and Soluble Water Contents}

Dry PAAm hydrogels were added to the biodiesel/diesel fuels samples and the proper contact 
between hydrogel particles and liquid phase was made by mild agitation. The swelling features of PAAm hydrogels were studied in a previous work (Fregolente et al., 2012). The quantity of PAAm (g) added in all experiments was fixed at $5.0 \mathrm{~g}$ in a fixed volume of oil sample of $100 \mathrm{~mL}$. After the process of removal of the water content, the hydrogel particles were recovered: the particles were filtered, re-dispersed in water and washed. Ethanol was used to dehydrate the hydrogel after the washing with water. The hydrogels particles were then dried in an oven at $70{ }^{\circ} \mathrm{C}$.

\section{RESULTS AND DISCUSSIONS}

\section{Humidity Absorption}

To evaluate the humidity absorption from the atmosphere, biodiesel and diesel samples were maintained in desiccators at constant relative humidities (RH) of $35,66,79$ and $87 \%$, at $20^{\circ} \mathrm{C}$. The humidity changes were monitored after 7 days under controlled conditions. After 7 days, the absorption of water from the humidity of the samples reached the corresponding equilibrium.

Changes in the absorbed water content (or released water content) of fuel samples were monitored in each case. Figure 1 shows the results of humidity content measurements (water content, in ppm) in four different conditions of $\mathrm{RH}$ for diesel fuel sample. Figure 2 shows the same experiment performed with biodiesel samples.

Figure 1 shows that the humidity content in the diesel samples increased progressively until stabilization after $96 \mathrm{~h}$ of exposition to $\mathrm{RH}$ of 66,79 and $87 \%$. The sample maintained at $87 \%$ RH obtained the maximum absorption of water, of $130 \mathrm{ppm}$, starting with a sample initially contained $86 \mathrm{ppm}$ of soluble water. The soluble water content increase for this last case was $55 \%$. For RH of 79 and $66 \%$ the highest levels of soluble water content absorbed by samples, after 72 hours of constant humidity exposition, were $111 \mathrm{ppm}$ and $100 \mathrm{ppm}$, respectively.

When the diesel fuel samples were exposed to $\mathrm{RH}$ of $35 \%$, the low relative humidity caused a gradual decrease in soluble water content in the samples until reaching the minimum of $65 \mathrm{ppm}$, after $144 \mathrm{~h}$ in a controlled atmosphere of $35 \%$ humidity. The decrease of soluble water content in diesel samples was $41 \%$.

Figure 2 shows the same experiment of humidity absorption realized for biodiesel samples. Following the same standard behaviour of absorption of soluble water content by diesel samples, Figure 2 shows that, for constant relative humidities of 66,79 and $87 \%$, the soluble water content in biodiesel samples increased after 24 hours of exposition.

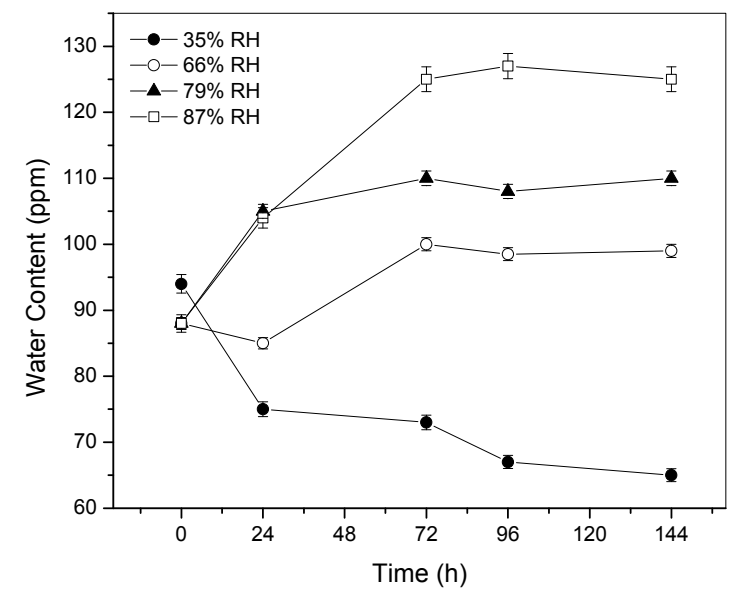

Figure 1: Absorption of water by diesel fuel at different constant relative humidities: $35 \%$ - •; $66 \%$ - o; $79 \%$ - А $; 87 \%$ - . Temperature of $20^{\circ} \mathrm{C}$.

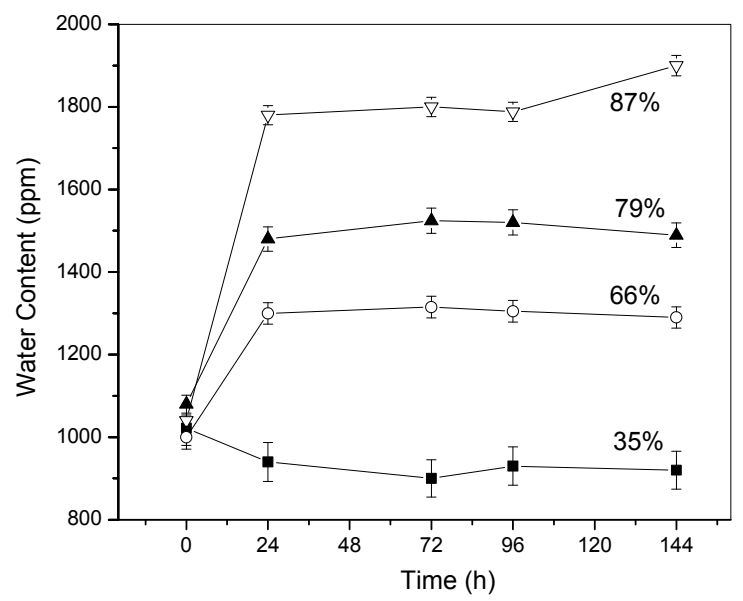

Figure 2: Absorption of water by biodiesel at different constant relative humidities: $35 \%-; 66 \%-0$; $79 \%-\boldsymbol{\Lambda} ; 87 \%-\nabla$. Temperature of $20^{\circ} \mathrm{C}$.

However, the absorption of soluble water was faster for biodiesel samples, reaching equilibrium in a reduced time, compared to the previous experiments. The increase of soluble water content in samples of biodiesel exposed at 87, 79 and $66 \% \mathrm{RH}$ were 70,50 and $32 \%$, respectively.

At $35 \% \mathrm{RH}$ the soluble water content in biodiesel decreased until the minimum of $833 \mathrm{ppm}$, starting from a biodiesel sample with $1145 \mathrm{ppm}$, presenting a $27 \%$ loss of soluble water of the biodiesel sample. These experiments simulate the behaviour of biodiesel and diesel fuel when they are stored or ex- 
posed to different weather situations of different relative humidities, such as rainy or dry days. In an environment with low $\mathrm{RH}(35 \%)$ there is loss of soluble water from the sample to the environment. The opposite was observed for samples stored in an environment with higher $\mathrm{RH}$.

\section{Experiments of Removal of Free and Soluble Wa- ter Content Using PAAm Hydrogels}

\section{Biodiesel Oil Samples}

Biodiesel has a polar chemical structure of carboxylate ester groups and presents the characteristics of absorbing more water than diesel. Thus, the addition of biodiesel to diesel increases the water retention capacity of the blend, as evidenced by previous data (Fregolente et al., 2010) of saturation curves of maximum water content of biodiesel and diesel blends at different temperatures. For instance, at $30^{\circ} \mathrm{C}$, the maximum saturated water content, or soluble water content, in B100 is $1840 \mathrm{ppm}$, whereas for $\mathrm{B} 20$ blend it is $270 \mathrm{ppm}$ and for B5 it is $150 \mathrm{ppm}$. For $\mathrm{B} 100$ at $20^{\circ} \mathrm{C}$, the maximum saturated water content is $1720 \mathrm{ppm}$ (soluble water content). Above this value of $1720 \mathrm{ppm}$, for $\mathrm{B} 100$ samples at $20^{\circ} \mathrm{C}$, the water present in B100 samples is considered to be free water. The saturation point is specified as the dashed line in Figures 3, 4, 5 and 6.

Dry hydrogels of PAAm were employed to remove the free and soluble water content of biodiesel samples. Figure 3 shows the reduction of soluble water content of biodiesel when dry PAAm hydrogels were added to the system. The biodiesel sample initially presented $1590 \mathrm{ppm}$ of soluble water (the biodiesel sample was not saturated with water). Note that the initial water content is situated above the dashed line. To consider the saturation of water content for biodiesel, a minimum of $1700 \mathrm{ppm}$ of water content is necessary. The high level of $1590 \mathrm{ppm}$ of water content was due to the exposition to the high $79 \% \mathrm{RH}$ for 10 days. Two samples were initially taken and the hydrogel was inserted into only one sample. The biodiesel sample without dry hydrogel was used to stipulate the control curve for the experiment. It was observed that, upon adding dry hydrogels, the reduction of water content was $49 \%$.

Figure 4 shows the removal of free and soluble water content of a saturated biodiesel sample. The addition of dry hydrogel to biodiesel samples (initial water content of $2100 \mathrm{ppm}$ ) resulted in the removal of free water and then the sequential removal of soluble water content. Figure 4 shows a control curve monitoring the water content of the sample without dry hydrogel. The control exhibited a mild reduction of water content due to the evaporation of the excess of free water content in the sample to the ambient at $65 \%$ RH. Adding dry hydrogel, the reduction of total water content in the biodiesel sample was $53.3 \%$.

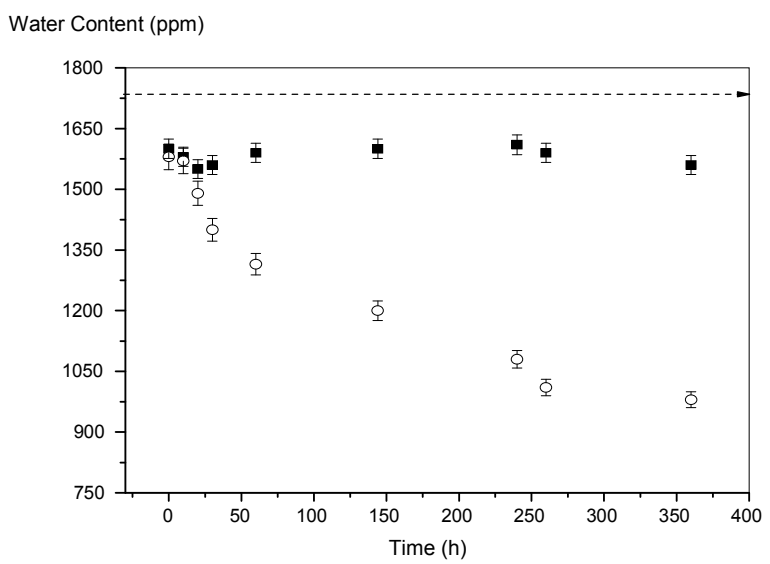

Figure 3: Removal of water content of a biodiesel sample (o) using dry PAAm hydrogel. Temperature $20^{\circ} \mathrm{C}$ and $\mathrm{RH}$ of $65 \%$. Reference sample ( ).

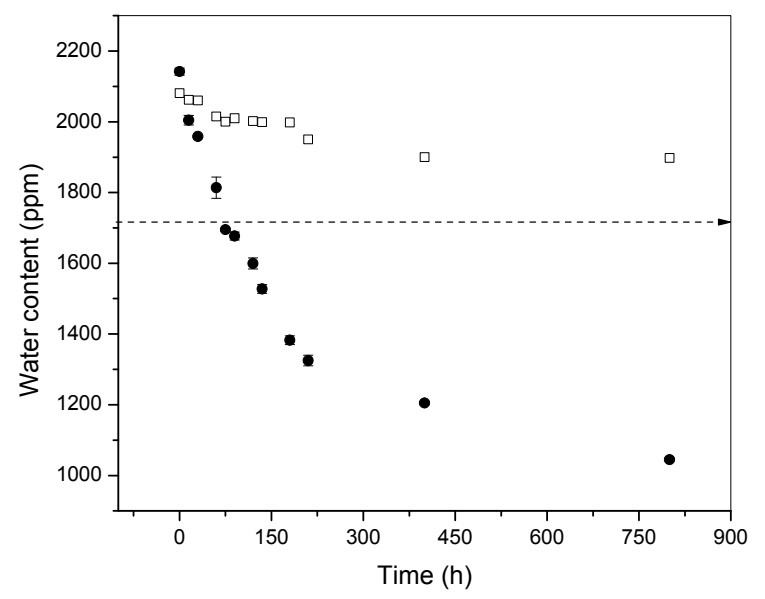

Figure 4: Removal of free and soluble water content of a biodiesel sample (•) using dry PAAm hydrogel. Temperature $20{ }^{\circ} \mathrm{C}$ and $\mathrm{RH}$ of $65 \%$. Reference sample ( ).

\section{Diesel Oil Samples}

Figure 5 shows the removal of the free and soluble water content of diesel oil samples using dry PAAm hydrogels as adsorbent materials. The control curve for this case also presented a mild decrease, due to the initial saturation of the diesel sample of free water content.

From a diesel oil sample initially containing 240 ppm, there was a reduction of $65.0 \%$ of total water content (free and soluble water content). 
Figure 6 shows two different curves for the decrease of water content using dry PAAm hydrogel: D1 is a non-saturated sample (only soluble water content) and D2 is a saturated diesel oil sample (without centrifugation, high free water content). There was a reduction of $80.4 \%$ in the water content of the D2 sample, due to the excess initial water content $(350 \mathrm{ppm})$ in the sample. There was a reduction of $32.0 \%$ in the water content of the D1 sample, initially with $98 \mathrm{ppm}$ of water content.

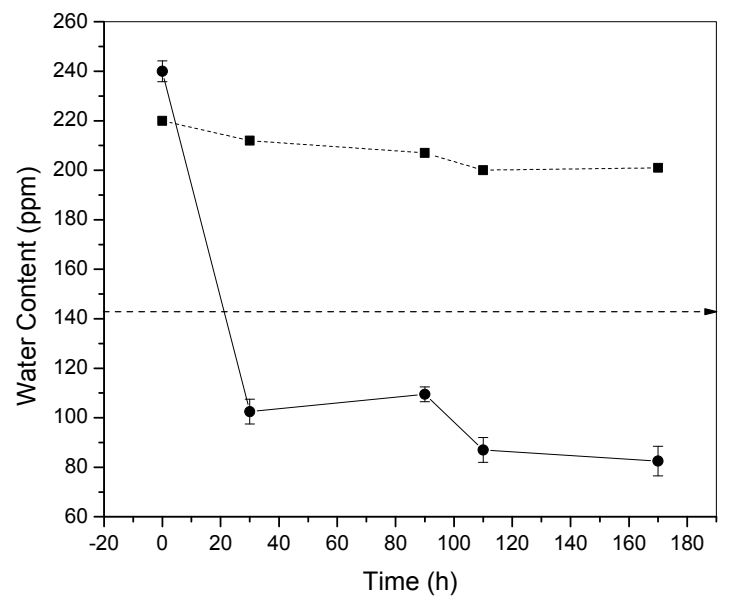

Figure 5: Removal of the free and soluble water content of a diesel oil sample $(\bullet)$ using dry PAAm hydrogel. Temperature $20{ }^{\circ} \mathrm{C}$ and $\mathrm{RH}$ of $60 \%$. Reference sample ( ).

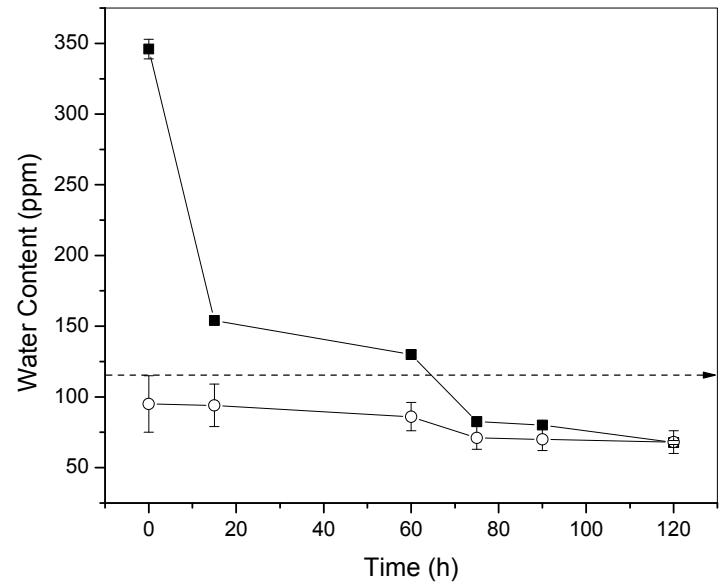

Figure 6: Removal of the free and soluble water of non-saturated D1 (o) and saturated D2 ( ) diesel oil samples using dry PAAm hydrogel. Temperature $30^{\circ} \mathrm{C}$ and $\mathrm{RH}$ of $60 \%$.

\section{Recovery of PAAm Hydrogels}

Even after several cycles of removal of water from fuels, cleaning and dehydration, the water retention capacity (swelling degree) of PAAm hydrogels was not affected. Throughout the duration of the research, the same hydrogels were reused and analyses of swelling degree were made periodically. After 10 months of use, swelling degree analyses were performed with results similar to those found after the synthesis of the hydrogel. After synthesis, the swelling degree was $15.40 \pm 1.36$ and after 10 months, it was maintained at $15.23 \pm 1.82$.

\section{CONCLUSIONS}

Hydrogel particles have been used successfully as an alternative method for the removal/reduction of water content of diesel and biodiesel fuels. Karl Fischer experiments showed that the treatment of saturated biodiesel with dry hydrogels reduced the water content by $53.3 \%$ from $2100 \mathrm{ppm}$. Treatment of non-saturated biodiesel with dry hydrogels reduced the soluble water content by $49.0 \%$. For saturated diesel fuel, the reduction of water content was $80.4 \%$ and for non-saturated diesel fuel the reduction was about $32.0 \%$. Based on the results obtained in this work, new hydrogels composed of different hydrophilic monomers will be synthesized and tested for their ability to remove water from fuel. The tests may yalso be extended to other liquid fuels such as gasoline and jet fuel. The goog performance of these hydrophilic hydrogel particles could be applicable for adsorbing water from other liquid fuel types and has the potential for development of new technologies for removing water from fuels.

\section{ACKNOWLEDGEMENTS}

The authors are grateful to FAPESP and CNPq for the financial support.

\section{REFERENCES}

ASTM Standards, D6751-03a, Specification for biodiesel fuel (B100) blend stock for distillate fuels. West Conshohocken, Penn., ASTM (2003).

Atadashi, I. M., Aroua, M. K., Abdul, A. R., Sulaiman, N. M. N., The effects of water on biodiesel production and refining Technologies: A review. Renewable and Sustainable Energy Reviews, 16, 346-3470 (2012).

Atadashi, I. M., Aroua, M. K., The effects of water on biodiesel production and refining technologies: A review. Renewable and Sustainable Energy Reviews, 16, 3456-3470 (2012). 
Bampi, M., Scheer, A. P., Castilhos, F., Application of near infrared spectroscopy to predict the average droplet size and water content in biodiesel emulsions. Fuel, 113, 546-552 (2013).

Bassil, M., Davenas, J., Tahchi, M. E., Electrochemical properties and actuation mechanisms of polyacrylamide hydrogel for artificial muscle application. Sensors and Actuators B, 134, 496-501 (2008).

Bücker, F., Santestevan, A. N., Roesch, L. F., Jacques, R. J. S., Peralba, M. C. R., Camargo, F. A. O., Bento, F. M., Impact of biodiesel on biodeterioration of stored Brazilian diesel oil. Int. Biodet. Biodegradation, 65, 172-178 (2011).

Deligkaris, K., Shiferaw, T., Olthuis, W., van der Berg, A., Hydrogel-based devices for biomedical applications. Sensors and Actuators, B, 147, 765774 (2010).

Fazal, M. A., Haseeb, A. S. M. A., Masjuki, H. H., Corrosion mechanism of copper in palm biodiesel. Corrosion Science, 67, 50-59 (2013).

Ferella, F., di Celso, G. M., De Michelis, I., Stanisci, V., Veglio, F., Optimization of the transesterification reaction in biodiesel production. Fuel, 89, 36-42 (2010).

Fregolente, P. B. L., Fregolente, L. V., Maciel, M. R. W., Maciel, R., Water content in biodiesel, diesel, and biodiesel-diesel blends. J. Chem. Eng. Data, 57, 1817-1821 (2012).

Fregolente, P. B. L., Wolf-Maciel, M. R., Water absorbing material to removal water from biodiesel and diesel. Procedia Engineering, 42, 1983-1988 (2012).
He, B. B., Thompson, J. C., Routt, D. W., Van Gerpen, J. H., Humidity absorption in biodiesel and its petro-diesel blends. Appl. Eng. Agric., 23, 7176 (2007).

Leung, D. Y., Koo, B. C. P., Guo, Y., Degradation of biodiesel under diferente storage conditions. Bioresource Technology, 97, 250-256 (2006).

Nur, H., Sowden, M. J., Corneliu, V. J., Mitchell, J. C., Harvey, P. J., Benee, L. S., Colloidal microgel in removal of water from biodiesel. Colloids and Surfaces A: Physicochem. Eng. Aspects, 335, 133-137 (2009).

Oliveira, M. B., Varanda, F. R., Marrucho, I. M., Queimada, A. J., Coutinho, A. P., Prediction of water solubility in biodiesel with the CPA equation of state. Ind. Eng. Chem. Res., 47, 42784285 (2008).

Perry, R. H., Green, D. W., Perry's Chemical Engineer's Handbook. 7th Ed. McGraw Hill, New York, 12-35 (1997).

Qunwei, T., Jihuai, M., Hui, S., Jianming, L., Shiun, F. H., Polyaniline/polyacrylamide conducting composite hydrogel with a porous structure. Carbohydrate Polymers, 74, 215-219 (2008).

Shah, P., Wee, C., White, J. M., Sanford, S., Meier, G., Experimental determination and thermodynamic modelling of water content in biodieseldiesel blends. Renewable Energy Group (2010).

Sørensen, G., Pedersen, D. V., Nørgaard, A. K., Sørensen, K. B., Nygaard, S. D., Microbial growth studies in biodiesel blends. Biores. Technol., 102, 5259-5264 (2011). 